\title{
Correction to: Success and complications of endotracheal intubation in critical care settings under COVID-19 protocols
}

\author{
Kia Dullemond ${ }^{1} \cdot$ Colby Renschler $^{1} \cdot$ Jan Trojanowski ${ }^{1} \cdot$ Frank Scheuermeyer $^{2,3} \cdot$ Rob Stenstrom $^{2,3}$. \\ Donald Griesdale $^{4} \cdot$ Ruth MacRedmond ${ }^{5}$. Elan Nattrass ${ }^{4} \cdot$ Lena Farina $^{5} \cdot$ Jeff Yoo $^{2}$
}

Published online: 22 March 2021

(c) Canadian Association of Emergency Physicians (CAEP)/ Association Canadienne de Médecine d'Urgence (ACMU) 2021

\section{Correction to: Canadian Journal of Emergency Medicine https://doi.org/10.1007/s43678-020-00061-z}

The original version of this article unfortunately contained a mistake. The following correction has therefore been made in the original: The spelling of Elan Nattrass' name was incorrect. The corrected author list is given above. The original article has been corrected.

The original article can be found online at https://doi.org/10.1007/ s43678-020-00061-z.

Frank Scheuermeyer

frank.scheuermeyer@gmail.com

1 Department of Emergency Medicine, Vancouver General Hospital and the University of British Columbia, Vancouver, BC, Canada

2 Department of Emergency Medicine, St Paul's Hospital and the University of British Columbia, 1081 Burrard St, Vancouver, BC V6Z 1Y6, Canada

3 Centre for Health Evaluation and Outcomes Sciences, Vancouver, BC, Canada

4 Division of Critical Care, Department of Medicine, Vancouver General Hospital and the University of British Columbia, Vancouver, BC, Canada

5 Division of Critical Care, Department of Medicine, St Paul's Hospital and the University of British Columbia, Vancouver, BC, Canada 